\title{
Complication avoidance in the treatment of malignant tumors of the skull base
}

\author{
James T. KryZanski, M.D., Donald J. Annino, JR., M.D., D.M.D., \\ and Carl B. Heilman, M.D. \\ Departments of Neurosurgery and Otolaryngology, Tufts-New England Medical Center, Boston, \\ Massachusetts
}

\begin{abstract}
The treatment of malignant skull base tumors has improved with the development of skull base surgical approaches that allow en bloc resection of a lesion and increase the efficacy of adjuvant therapies. The anatomical complexity of these lesions and their surroundings has led to a relatively high complication rate. Infection and cerebrospinal fluid fistulas are the most common serious procedure-related complications. They result from the frequent necessity of working in a contaminated space such as the paranasal sinuses as well as from the creation of large dural and skull base defects. The authors have reviewed the literature regarding complications of surgery for malignant skull base lesions and present several techniques and strategies for minimizing their incidence by performing the craniofacial approach to anterior skull base lesions.
\end{abstract}

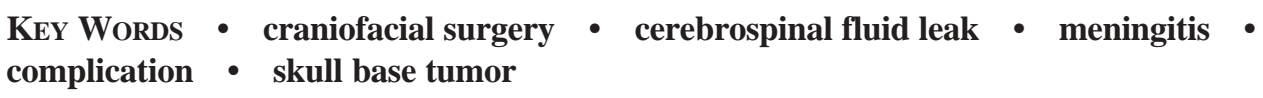

The development of skull base surgical approaches and their increasing use have improved treatment of malignant tumors of the skull base. Skull base approaches have allowed for successful resection of many tumors once considered inoperable. Skull base tumors present numerous surgery-related problems because of involvement of functional structures, difficult access, and the creation of large defects after removal. These surgical challenges have led to relatively high complication rates in skull base surgical series. For many reasons, malignant skull base tumors are among the most prone to surgery-related complications. Our goal is to present a review of procedure-related complications arising from skull base malignancies and to offer strategies for their avoidance.

\section{REVIEW OF TREATMENT OF MALIGNANT SKULL BASE TUMORS}

In the preoperative period, proper patient selection and delineation of surgery-related goals are the most important elements in reducing complications associated with the procedure. Malignant skull base disease is heterogeneous, often involving uncommon tumors, as reflected by the number of patients with malignant skull base tumors treat-

Abbreviation used in this paper: CSF = cerebrospinal fluid. ed at our center since 1994 (Table 1). Multimodality treatment is often indicated, and the proper use and timing of adjunctive therapies is a critical consideration in the management of a patient with a skull base tumor. In addition, a biopsy sample should be obtained when possible prior to resection so that surgery can be planned in light of the known behavior of the lesion.

Several radiographic features necessitate preoperative consideration, particularly when gross-total resection is the goal (Table 2). Orbital involvement of the malignant process may require enucleation of the eye, or it may risk visual deficit if the eye is to be preserved. The incidence of postoperative CSF leakage and meningitis correlates with involvement of the dura and brain. The risk of CSF leakage and infection are also increased in processes involving the middle ear and/or eustachian tube. When the lesion involves major arteries or dural sinuses, the risks of intra- and postoperative hemorrhage as well as arterial or venous infarction are present. Proximity or involvement with cranial nerves and/or the cavernous sinus will greatly increase the risk to these structures. Preoperative radiography can typically identify the structures and potential complications of particular significance for a given lesion. When planning surgery, the surgeon must then focus on minimizing the occurrence of these complications. An example of a sinonasal carcinoma involving significant anterior skull base and intradural invasion is shown in Fig. 1. 


\section{J. T. Kryzanski, D. J. Annino, Jr., and C. B. Heilman}

TABLE 1

Malignant skull base lesions treated at Tufts-New England Medical Center since 1994

\begin{tabular}{lc}
\hline \hline \multicolumn{1}{c}{ Type of Lesion } & No. Treated \\
\hline adenoid cystic carcinoma & 2 \\
carcinoma ex pleomorphic adenoma & 1 \\
chondrosarcoma & 6 \\
chordoma & 4 \\
carcinoma & 3 \\
esthesioneuroblastoma & 3 \\
fibrous histiocytoma & 1 \\
invasive angiofibroma & 1 \\
lymphoma & 1 \\
malignant meningioma & 1 \\
metastasis & 3 \\
nasoethmoid adenocarcinoma & 1 \\
nasoethmoid sarcoma & \\
$\quad$ fibrosarcoma & 2 \\
$\quad$ leiomyosarcoma & 1 \\
$\quad$ sarcoma ex paget & 1 \\
rhabdomyosarcoma & 1 \\
osteoblastoma & 1 \\
pituitary carcinoma & 1 \\
plasmacytoma & 1 \\
sinonasal undifferentiated carcinoma & 1 \\
\hline
\end{tabular}

\section{SURGERY-RELATED COMPLICATIONS}

Surgery for malignant skull base lesions is plagued by the same complications associated with any skull base procedure, although the incidence is generally higher. The higher complication rate is a result of the increased invasiveness of malignant lesions, poorer condition of the patient, need for multiple surgeries, and adjuvant therapies such as radiotherapy. The only recent series of malignant skull base lesions comprising all locations is that reported by Donald, ${ }^{2}$ in which 107 consecutive procedures performed in 98 patients were associated with an overall complication rate of $50.5 \%$. The surgery-related mortality was $4 \%$, half of which was attributable to vascular causes. Donald reported a CSF leakage rate of $11.2 \%$, a $15 \%$ incidence of wound breakdown, and a $4.8 \%$ incidence of meningitis. Notably, all cases of purulent meningitis occurred in patients with postoperative CSF leaks. There was a trend toward a higher complication rate in those who had undergone middle cranial fossa surgery and a statistically significant increase in those in whom prior surgery had been performed. Surprisingly, he found that a history of radiotherapy did not increase the overall complication rate.

TABLE 2

Radiographic features that predict surgery-related complications

orbital involvement

dural \& brain invasion

cribriform plate involvement

cavernous sinus invasion

proximity to cranial nerves

dural sinus involvement

arterial invasion or encasement

proximity to eustachian tube

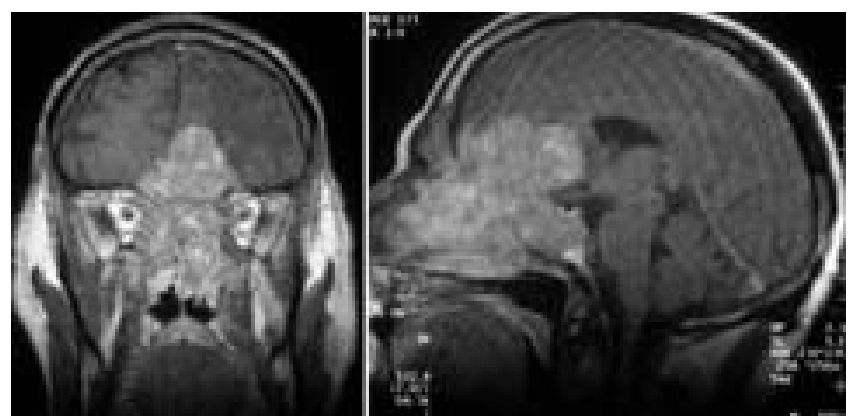

Fig. 1. Postcontrast magnetic resonance images. Left:Coronal image obtained in a patient with sinonasal carcinoma and intradural extension. Right: Sagittal image.

The most extensive anatomical site-specific literature concerning complications of surgery for malignant skull base lesions is found regarding the craniofacial resection of malignant anterior skull base lesions. Whereas many malignant skull base tumors require a tailored approach, anterior skull base malignancies are usually treated in a relatively standard fashion by performing graded craniofacial resection. Therefore, comparison of various techniques and their associated complications is possible. Kraus, et al., ${ }^{5}$ described a series of 86 patients who underwent craniofacial resection of malignant tumors and reported an overall complication rate of 39\%. These included a surgery-related mortality rate of $2 \%$, a wound infection or osteomyelitis rate of $21 \%$, and a CSF leakage rate of $2 \%$. They found that patient age greater than 60 increased the risk of complications but not factors such as previous surgery or radiotherapy. Dias, et al., ${ }^{1}$ reported a series of 104 patients with benign and malignant lesions who underwent craniofacial resection. Their overall complication rate was $48.6 \%$, and the surgery-related mortality rate was $7.6 \%$. Notably, seven of the eight deaths resulted from meningitis associated with CSF leakage. Local septic conditions accounted for $54.7 \%$ of all complications. They found the occurrence of CSF leakage to correspond to both invasion of the dura and the absence of a pericranial or galeal-pericranial reconstruction. There are several other reports in which the authors have reported complication rates similar to those in the aforementioned series for craniofacial resections. ${ }^{3,8,9}$

Manolidis, et al., ${ }^{6}$ have published the largest review of strictly temporal bone and lateral skull base malignancies. They reported a major complication rate of $21 \%$. This does not include expected cranial neuropathies. They found a surgery-related mortality rate of $4 \%$, a CSF leakage rate of $6 \%$, and a rate of major wound complications of $7.4 \%$. In summary, those who have published the most recent series are relatively consistent in reporting surgeryrelated complication rates of 30 to $50 \%$ when performing resection of malignant skull base lesions. The complication rate does not seem to correlate with the location of the lesion but does increase in cases in which the lesion has been previously treated. Complications of CSF leakage and infections usually account for over half of all complications. The rate of surgery-related mortality varies from 2 to $7 \%$, and death was most often caused by vascular or infectious complications, particularly meningitis. 


\section{COMPLICATION AVOIDANCE}

It is clear from the previous discussion that resectionrelated complication rates in cases of malignant skull base lesions are significant. Minimizing these complications demands careful planning and meticulous technique. In addition, there is considerable variation in practice among skull base centers concerning many of the crucial steps in a given procedure. We will discuss our techniques for complication avoidance in malignant skull base lesions via the craniofacial approach; the latter route is used as a paradigm because the anterior skull base is the area most commonly affected by malignancy and the complications of this approach have been the most extensively reported. Most of the key points and principles, however, are applicable to any malignant skull base procedure.

As previously discussed, the vast majority of serious complications in anterior skull base surgery result from infection and/or CSF leakage. Regarding infection, there are several key steps that we believe can help to minimize the risk of postoperative abscess, bone flap infection, and/or meningitis. First, the development of an intact anterior-based pericranial flap is essential so that the intracranial cavity is sealed from the nasal cavity with live tissue. The flap must be long enough to cover completely the entire anterior skull base defect and extend posterior to the suture line of the primary dural repair (Video Clip 1).

Click to view Video Clip 1: The clip shows the development of an anterior based pericranial flap.

We prefer to reconstruct the anterior skull base by using two layers of tissue for closure as well as an intervening structural support (Fig. 2). The first tissue layer is the primary dural repair, and the second is the vascularized anterior-based pericranial flap. The details regarding the structural support will be discussed later. Second, when the surgical exposure involves a contaminated space, we routinely complete the intradural portion of the procedure and then seal the dura prior to entering the contaminated

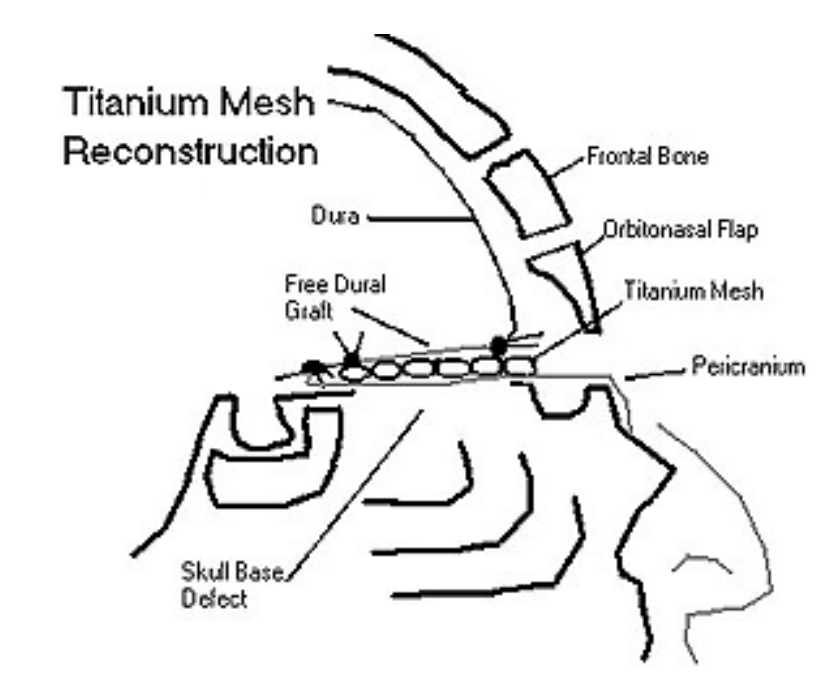

Fig. 2. Schematic depicting anterior skull base reconstruction in which there is a vascularized pericranial flap directly over the defect, titanium mesh over the pericranium, and a dural patch. space. For example, during a craniofacial approach, we first dissect the intradural tumor from the brain and define the dural margins around the tumor (Video Clip 2). We then primarily repair the dural defect prior to entering the nasal cavity (Video Clip 3). The use of an orbitonasal bar osteotomy necessitates entry into the frontal sinus. Because the frontal sinus is usually sterile, we do operate within the frontal sinus space while the dura is being primarily repaired; however, we try to not enter the ethmoid sinuses, nasofrontal ducts, or nasal cavity until the intradural space has been isolated. This reduces contamination of the intradural space. In our experience there have been no related brain abscess complications. This will occasionally compromise the ability to perform a strict en bloc resection because the intracranial portion of the lesion may need to be partially removed to allow visualization and suturing of the dural margin at the posterior aspect of the lesion.

Click to view Video Clip 2: Dissection of the tumor from dura
is demonstrated.
Click to view Video Clip 3: The clip shows the technique for
dural patch repair.

The primary dural repair must be meticulous. This cannot be overemphasized. We favor a free pericranial graft obtained from the parietal skull behind the bicoronal incision for performing the primary dural repair (Video Clip 4). This autologous tissue is probably vascularized relatively rapidly and therefore acts for the least amount of time as a potential nidus of infection. If free pericranium is not available, allografts such as alloderm or cadaveric fascia lata are alternatives. The posterior dural margin along the tuberculum or planum sphenoidale is usually the most difficult to repair because the dura may be friable or the cuff between the defect and the optic nerves may be very small. We use multiple interrupted sutures in this region to achieve the best possible result (Fig. 3 and Video Clip 5).

Click to view Video Clip 4 The harvesting of the free parietal pericranial graft is depicted.

Click to view Video Clip 5 The clip provides a closeup of the sutured posterior dural cuff.

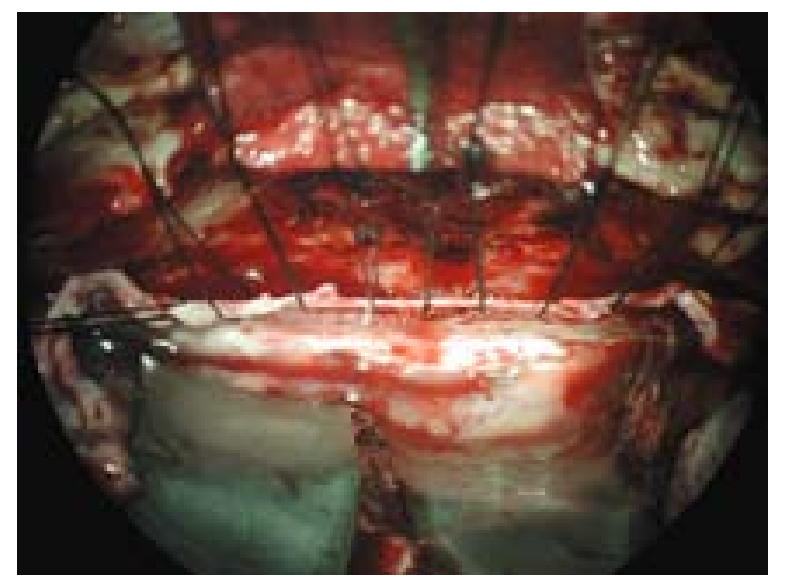

Fig. 3. Intraoperative photograph demonstrating the interrupted sutures in the posterior dural margin. 
In infection avoidance other important issues are the management of the frontal sinus and the location of the vascularized pericranial flap relative to the bone flaps. Cranialization of the frontal sinus must be thorough, and mucosal remnants must be drilled off all remaining sinus walls to prevent mucocele formation (Video Clip 6). We believe that the bone flaps should be replaced in a manner that minimizes their exposure to contaminated areas. We perform craniofacial resections by using a two-piece craniotomy: a bifrontal flap and a separate orbitonasal bar (Fig. 4). The removal of a bifrontal bone flap provides excellent visualization of the frontal dura beneath the orbital bar, such that unwanted dural lacerations are unlikely during osteotomy of the orbitonasal bar (Video Clip 7). The lateral osteotomies of the orbitonasal bar are beveled toward the midline, and this prevents subsidence of the orbital bar over time or in the event that the forehead sustains traumatic injury. In addition, we typically make a relatively short orbitonasal bar osteotomy. The lateral orbital rims usually do not have to be removed. Our osteotomy cuts often start at the supraorbital notch and slant toward the midline. If the orbitonasal bar ever had to be removed because of a postoperative wound infection, the cosmetic effect would be less severe and the defect easier to repair than if the orbitonasal bar osteotomy encompassed the entire orbital rims bilaterally. Infection of these devitalized bone flaps has been a significant problem in the reviewed series. When reconstructing the skull base, the anterior pedicled pericranial flap harvested during the opening is placed over the skull base defect and tacked to the dura posterior to the primary dural repair. This provides the primary barrier between the nasal and intracranial cavities. We place the pericranial flap beneath

Click to view Video Clip 6 Cranialization of the frontal sinus is shown.

Click to view Video Clip 7 The location of the bifrontal craniotomy is indicated.

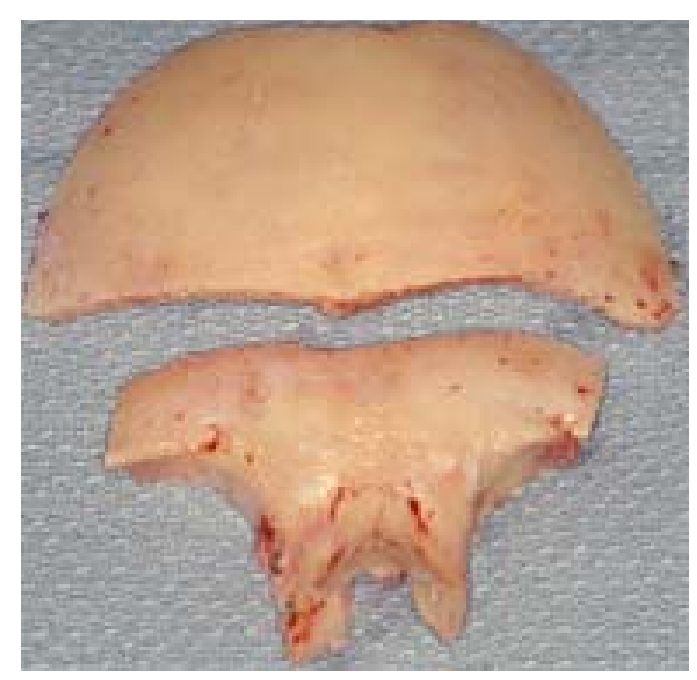

Fig. 4. Photograph showing the two bone flaps used in the craniofacial approach. Currently our orbitonasal flap includes less of the nasal bone than shown here because it is then easier to place the pericranial pedicle beneath the orbitonasal flap. the orbitonasal bar whenever possible to reduce the exposure of the bone flaps to the nasal cavity (Video Clip 8). This limits the amount of the nasal bone that can be removed with the orbital nasal bar. If the nasal bones are removed along with the orbital bar, it is difficult to get the pericranial graft to fold nicely into the surgical defect. When placing the pericranial graft beneath the orbital bar, however, it is important not to compress the pericranium where it passes beneath the orbitonasal bar. Compression can generate edema in the intracranial portion of the pericranium, occasionally causing severe swelling of the flap and requiring surgical removal. ${ }^{4}$ The osteotomy cuts in the medial orbital roof directly under the orbital rim can be widened slightly to allow space for the pericranial graft to pass. Widening the osteotomy cuts in this area is not cosmetically apparent.

Click to view Video Clip 8: The clip demonstrates the final placement of bone flaps and pericranium.

In several of the reviewed series, meningitis was the most deadly complication associated with craniofacial resection for malignant lesions. Cerebrospinal fluid leakage is the most significant risk factor for meningitis and a remarkably consistent complication reported in every reviewed series. The single most important factor involved in reducing the incidence of CSF leakage has already been discussed: a meticulous dural closure. In our opinion, relying on fibrin glue to seal the dura instead of tightly placed interrupted sutures is rarely successful. Dural closure, however, is not the only consideration when attempting to prevent CSF fluid leakage. We believe that mechanical reconstruction of large $(>1.5-\mathrm{cm}$-wide) anterior skull base defects helps to prevent CSF leakage by reducing tension on the repaired dura. Our preference is to perform mechanical reconstruction by using either vascularized outer table bone or titanium mesh contoured to cover the entire defect (Video Clip 9). In using vascularized outer table bone the skull base reconstruction is provided with exclusively vitalized tissue, but the procedure is labor intensive and creates a frontal bone outer table defect. The use of titanium mesh is faster and the material is easier to contour to the skull base and orbital roofs. When using the latter, we prefer to place the mesh above the vascularized pericranial flap so that the mesh is separated from the nasal cavity. This is analogous to treatment of the orbitonasal bar. Although the necessity of mechanical reconstruction is debatable, we believe that it eliminates tension on the margins of the repaired dura by preventing distension of the dural graft into the anterior skull base defect.

Click to view Video Clip 9: The shape and placement of the titanium mesh reconstruction are demonstrated.

The last option in the prevention of CSF leakage is the judicious use of spinal drainage. Spinal drainage is not a risk-free procedure, however, and has been associated with a 59\% incidence of minor complications (for example, headache and nausea) and a $12.5 \%$ incidence of major complications, including vocal cord paralysis and posterior cerebral artery infarction from transtentorial herniation. ${ }^{7}$ Thus we therefore do not perform prophylactic spinal drainage in all patients; rather it is only used in those 
judged to be at a very high risk for CSF leakage or those in whom a postoperative CSF leak has been demonstrated.

Regarding tumor removal, en bloc resection particularly of the skull base and facial component is the goal. This is usually performed via anterior skull base osteotomies around the tumor such that the tumor and a small margin of bone are resected (Fig. 5 left). The anterior skull base osteotomies are performed using a diamond-burr drill instead of an osteotome, and we believe that this helps minimize the risk to adjacent structures (Video Clip 10). The facial component of the tumor is then dissected free and the tumor removed (Video Clip 11). The resulting anterior skull base defect is shown in Fig. 5 right.

Click to view Video Clip 10: The clip shows the skull base osteotomies around the tumor.

Click to view Video Clip 11: Tumor removal is demonstrated.

Although not usually considered with complications, restoration of cosmesis deserves discussion because it relates to the patient's postoperative quality of life. Patients with malignant skull base lesions often undergo adjuvant radiotherapy that may thin the scalp and make cranial defects or hardware more apparent. We drill the screw holes for the titanium miniplates prior to performing the craniotomy and orbital osteotomy so that perfect repositioning of the bone flaps is obtained during reconstruction (Fig. 6). Additionally, we often use hydroxyapatite bone cement to fill the defects around the edges of bone flaps, particularly in women with a thin forehead scalp.

\section{CONCLUSIONS}

Surgery for malignant lesions of the skull base is associated with a high complication rate. The most frequent complications include infection and CSF leakage. We describe our strategies for avoiding these complications; the anterior craniofacial resection is used as a paradigm. Because our techniques have evolved over the past few years, our current series in which they are applied is too small to demonstrate a lower complication rate. Nevertheless, we are optimistic that these techniques will prove useful. The key points can be summarized as follows:

1) An anterior-based pericranial flap of adequate length is created.

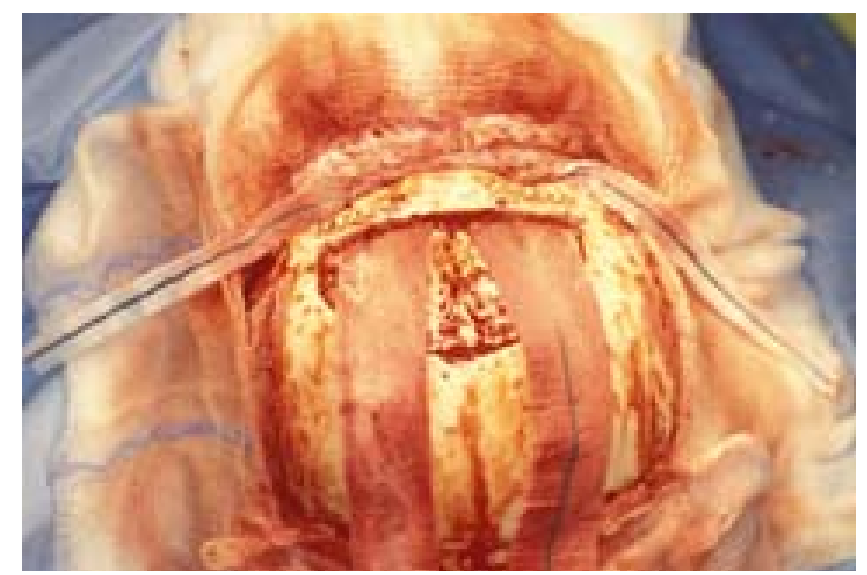

Fig. 6. The bifrontal craniotomy has been performed. The miniplates for the orbitonasal bar are fitted and holes drilled prior to its removal.

2) Low exposure of the anterior skull base is created by a bifrontal bone flap and a narrow orbitonasal bar bone flap with beveled edges; miniplate screw holes are drilled prior to making the osteotomy cuts.

3) Complete cranialization of the frontal sinus is performed to prevent mucocele formation and allow the pericranial graft to adhere to the residual walls of the frontal sinus floor.

4) The intradural space is isolated by primary dural repair prior to working in a contaminated cavity such as the ethmoid sinuses, nasofrontal ducts, or nasal airways. A free pericranial graft obtained from the parietal skull is a good option for primary dural repair.

5) The CSF is separated from the nasal cavity by using two layers of tissue; the dural repair is the first layer and the vascularized pericranial flap is the second.

6) The vascularized pericranial flap should separate all bone flaps from the nasal cavity (that is, the pericranial flap should go beneath the orbitonasal bar if possible).

7) Meticulous dural suturing should be performed rather than relying on fibrin glue.

8) Mechanical reconstruction of large anterior skull base defects should be conducted using titanium mesh or vas-

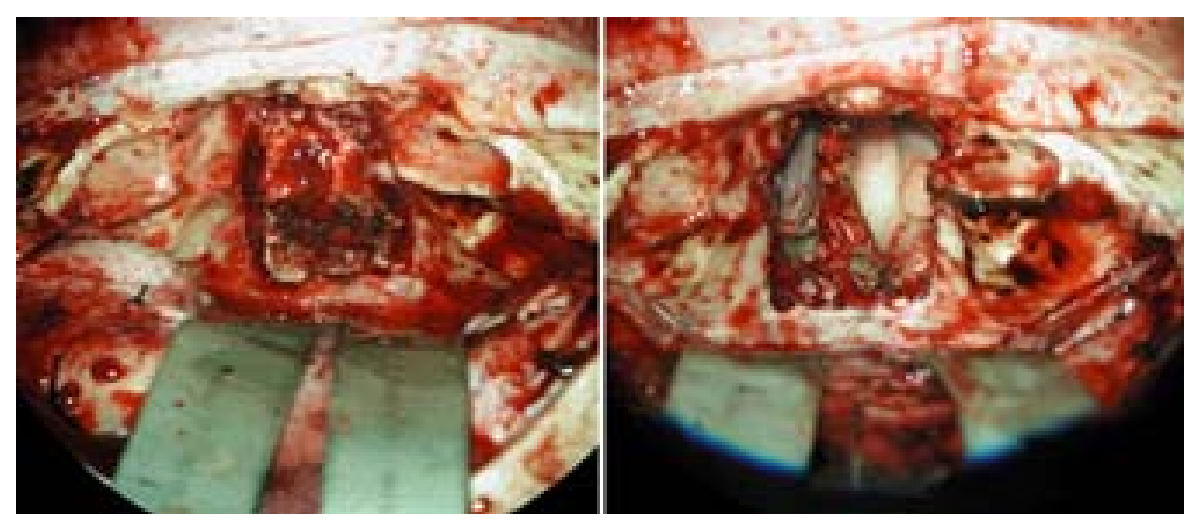

Fig. 5. Intraoperative photograph. Left: Anterior skull base with osteotomies made around the tumor. Right: Anterior skull base defect after tumor removal. 


\section{J. T. Kryzanski, D. J. Annino, Jr., and C. B. Heilman}

cularized outer table bone; this prevents traction on the suture line of the dural repair.

9) Spinal drainage should be performed judiciously and routinely used only in patients in whom CSF leaks occur postoperatively.

We have used the craniofacial resection as a framework for discussing these techniques and principles because the anterior skull base is commonly affected by malignant lesions. We believe that most of these principles apply to malignant tumors in other skull base regions as well. Skull base malignancies are among the most difficult cranial tumors to remove. Some of our recommendations are subjects of current debate and not proven in the literature. Nevertheless, we have noticed a decrease in our complication rate since we began using these techniques.

\section{References}

1. Dias FL, Sa GM, Kligerman J, et al: Complications of anterior craniofacial resection. Head Neck 21:12-20, 1999

2. Donald PJ: Complications in skull base surgery for malignancy. Laryngoscope 109:1959-1966, 1999

3. Fliss DM, Zucker G, Cohen A, et al: Early outcome and complications of the extended subcranial approach to the anterior skull base. Laryngoscope 109:153-160, 1999
4. Jensen R, McCutcheon IE, DeMonte F: Postoperative swelling of pericranial pedicle graft producing intracranial mass effect. Report of two cases. J Neurosurg 91:124-127, 1999

5. Kraus DH, Shah JP, Arbit E, et al: Complications of craniofacial resection for tumors involving the anterior skull base. Head Neck 16:307-312, 1994

6. Manolidis S, Pappas D Jr, Von Doersten P, et al: Temporal bone and lateral skull base malignancy: experience and results with 81 patients. Am J Otol 19 (Suppl 6):S1-S15, 1998

7. Roland PS, Marple BF, Meyerhoff WL, et al: Complications of lumbar spinal fluid drainage. Otolarygol Head Neck Surg 107:564-569, 1992

8. Salvan D, Julieron M, Marandas P, et al: Combined transfacial and neurosurgical approach to malignant tumors of the ethmoid sinus. J Laryngol Otol 112:446-450, 1998

9. Shah JP, Kraus DH, Bilsky MH, et al: Craniofacial resection for malignant tumors involving the anterior skull base. Arch Otolaryngol Head Neck Surg 123:1312-1317, 1997

Manuscript received March 25, 2002.

Accepted in final form April 30, 2002.

Address reprint requests to: Carl B. Heilman, M.D., Department of Neurosurgery, Tufts-New England Medical Center, 750 Washington Street, Boston, Massachusetts 02111. email: cheilman@ lifespan.org. 